\title{
Central Obesity and Health-related Factors among Middle-aged Men: a Comparison among Native Japanese and Japanese-Brazilians Residing in Brazil and Japan
}

\author{
Andiara Schwingel $^{1)}$, Yoshio Nakata ${ }^{1), 2)}$, Lucy S. Ito ${ }^{3), 4)}$, Wojtek J. Chodzko-Zajko ${ }^{5)}$, \\ Christopher T. Erb $^{5)}$, Ryosuke Shigematsu' ${ }^{6}$, Sueli M. Oba-Shinjo ${ }^{3)}$, Tomoaki Matsuo ${ }^{1)}$, \\ Samuel K. Shinjo ${ }^{4)}$, Miyuki Uno ${ }^{3)}$, Suely K. N. Marie ${ }^{3)}$ and Kiyoji Tanaka ${ }^{1), 2)}$ \\ 1) Graduate School of Comprehensive Human Sciences, Sports Medicine, University of Tsukuba \\ 2) Center for Tsukuba Advanced Research Alliance, University of Tsukuba \\ 3) Department of Neurology, School of Medicine, São Paulo University, Brazil \\ 4) Japanese Brazilian Health Professional Volunteer Group, Brazil \\ 5) Department of Kinesiology and Community Health, University of Illinois, USA \\ 6) Faculty of Education, Mie University
}

\begin{abstract}
The objective of this study was to investigate the influence of different cultural environments on the development of obesity by examining the association of central obesity, lifestyle, and selected coronary risk factors among people with identical Japanese genetic backgrounds living in Japan and Brazil. One hundred and four native Japanese and 286 Japanese-Brazilians residing in Brazil and Japan aged 35 years or over were studied. Obesity, metabolic risk factors for coronary disease, and history of regular sports activity, daily physical activity, and eating habits were assessed. The results showed Japanese-Brazilians residing in Brazil with significantly higher waist circumference values, and greater prevalence of central obesity compared to native Japanese and JapaneseBrazilians residing in Japan. The risk of developing central obesity was found to be 2.8 times higher among JapaneseBrazilians residing in Brazil. However, this association was no longer found to be significant after adjusting for lifestyle factors in the logistic model. Additionally, waist circumference was found to be significantly associated with metabolic risk factors for coronary disease. These findings suggest substantial variation in measures of central obesity among the three groups of Japanese ancestry, and underscore the heterogeneity of risk factors among communities of Japanese ancestry living in different cultural environments. The results also suggest that immigrant men exposed to the Brazilian cultural environment are more susceptible to the development of central obesity, and it seems to be associated with various lifestyle items and metabolic risk factors for coronary disease. $J$ Physiol Anthropol 26(3): 339-347, 2007 http://www.jstage.jst.go.jp/ browse/jpa2
\end{abstract}

[DOI: 10.2114/jpa2.26.339]

Keywords: Japanese-Brazilian, Central obesity, Cultural environment, Immigration, Coronary risk factor, Lifestyle

\section{Introduction}

Obesity is a condition that represents a serious worldwide threat to public health and has been found to increase the risk of various comorbidities, including coronary heart disease and type-2 diabetes (Haslam and James, 2005). Body mass index (BMI) is often used to reflect total body fat amount, while waist circumference (WC) is used to measure the degree of central fat distribution. Central distribution of body fat is an indicator of abdominal visceral fat, which has been found to be an important predictor of cardiovascular morbidities in men; therefore, it is thought to be a more specific risk factor for health problems than BMI alone (Japan Society for the Study of Obesity, 2002; Kobayashi et al., 2001; Lakka et al., 2001; Zhu et al., 2005).

Epidemiological studies have demonstrated that lifestyle choices, accumulated across the life course, greatly influence the risk of developing obesity (Haslam and James, 2005). Greater consumption of high-fat and energy-dense food is generally considered to be associated with the increasing prevalence of obesity (Howarth et al., 2006; Schrauwen and Westerterp, 2000). Also, increases in energy expenditure through sports and other forms of physical activity have been reported as important lifestyle factors that enhance overall 
health and prevent the development of central obesity (Jakicic and Otto, 2006; Kay and Fiatarone Singh, 2006; Miyatake et al., 2002). Another contributory factor for the development of obesity is an individual's genetic background. Large differences in prevalence of obesity have been reported across different ethnic groups (McNeely et al., 2001; Tanaka et al., 2003; World Health Organization, 2000a).

Obesity was reported to be associated with the development of a number of chronic diseases in the Japanese population, even within relatively mild levels of obesity (Kanazawa et al., 2002). For instance, studies of Japanese immigrants in the United States report a high prevalence of both obesity and several risk factors for obesity-related diseases compared to native Japanese (Curb and Marcus, 1991; Miura et al., 2006; Nakanishi et al., 2004; Yano et al., 1988). Additionally, changes in disease patterns have been observed in successive generations of people of Japanese ancestry residing in Brazil, suggesting that factors associated with the migration process and cultural environment may influence the risk of developing some diseases (Cardoso et al., 1997; Ferreira et al., 2002; Gimeno et al., 2005; Iunes et al., 1994; Tsugane et al., 1995).

Japan and Brazil have experienced two major immigration movements. First was Japanese immigration to Brazil, which began in 1908, and generated a community of approximately 1.3 million people of Japanese ancestry living in Brazil. More recently (beginning about 20 years ago) there has been a movement of back-immigration to Japan, which resulted in a community of nearly 300,000 Japanese-Brazilians living in Japan. Considering the great number of Japanese immigrants, there is little information available in the literature concerning risk factors for developing central obesity and its health consequences among Japanese-Brazilian men. Therefore, this study investigated the influence of different cultural environments (Japan and Brazil) on the development of obesity by examining the association of central obesity, selected coronary risk factors, and lifestyle characteristics, such as sports activity, physical activity in daily life, and nutritional habits among people with identical Japanese genetic backgrounds. This investigation clarifies our understanding of the impact of cultural environment on a number of important chronic disease and lifestyle characteristics known to be important for public health.

\section{Methods}

\section{Study population}

The present study was undertaken in 2004 and 2005 to investigate the health status and health-related behaviors of people aged 35 years and over in urban and suburban areas of Japan and Brazil. This study involved native Japanese and Japanese-Brazilians, with both parents of Japanese ancestry, who were raised in Brazil. Participants of this study were volunteers in apparently good health. Thirty-eight participants were excluded from the analysis (both parents were not of Japanese ancestry in 30 cases, and 8 cases were excluded due to problems with data collection). In total, data from 390 men ranging in age from $35-79$ years $(53.7 \pm 10.4 \mathrm{yr})$ were included in the study. Among those were 104 native Japanese (J-J group) residing in the central area of Japan (Kanto). None of the participants in this group had left the country for more than three consecutive months during their lifetime. Additionally, 178 Japanese-Brazilians residing in Japan (JB-J group) were included in the study. The majority of participants in this group were from the second generation of Japanese descent (Table 1). At the time of data collection, all participants in this group had been living in Japan (Kanto area) for a length greater than one year, with $59.6 \%(n=106)$ living in Japan for a period greater than five years. Finally, 108 Japanese-Brazilians residing in São Paulo city, Brazil, (JB-B group) were enrolled in this study. The majority of participants in this group were from the second generation of Japanese descent, as shown in Table 1.

\section{Protocol}

The overall protocol was approved in Brazil by the Ethical Committee of the School of Medicine, University of São Paulo, and in Japan by the Human Investigation Review Committee at the School of Comprehensive Human Sciences, University of Tsukuba. A statement of informed consent (available bilingually in Japanese and Portuguese) was obtained from all participants prior to initiation of the data collection. Data collection was carried out for all groups during mild seasons (spring or autumn), in order to avoid the influence of climatic factors in the variables measured for this study. The survey consisted of both quantitative clinical examinations and qualitative information assessed through self-administered questionnaires checked by trained researchers.

Data collection was conducted in the morning, following a period of at least 12 hours in which no food or medication was taken by the participants. A sample of venous blood was drawn from each participant and collected in vacuum tubes. The blood samples were stored at $4^{\circ} \mathrm{C}$ until they were delivered to the laboratory for analysis (Kotobiken Medical Laboratories, Inc., Tsukuba, Japan). For samples collected in Japan, all analyses were conducted within 72 hours of the blood collection. For samples collected in Brazil, blood tubes were immediately centrifuged to obtain plasma and serum, and frozen at $-80^{\circ} \mathrm{C}$. After data collection in Brazil, frozen blood samples were transferred to Japan to be analyzed by the same procedure in the same laboratory. Serum total cholesterol was measured by the cholesterol oxidase HDAOS method (Wako Pure Chemical Industries, Ltd.), high-density lipoprotein (HDL) cholesterol using a modified enzymatic method (Kyowa Medex Co., Ltd.), and triglycerides by a GPO-HDAOS method, a glycerol blanking method assay (Daiichi Pure Chemicals Co., Ltd.). The low-density lipoprotein (LDL) cholesterol was calculated according to the method of Friedewald et al. (1972). A homogeneous method based on an innovative detergent technology (A\&T Corporation) was assessed for fasting plasma glucose. 
Systolic and diastolic blood pressure was measured in the sitting position after 10 minutes of rest using an automatic device (Omron HEM-762, expressed as $\mathrm{mmHg}$ ). Weight to the nearest $0.1 \mathrm{~kg}$ was measured using a digital scale balance (TBF-551, Tanita, Tokyo, Japan) and height to the nearest 0.1 $\mathrm{cm}$ using a wall-mounted stadiometer (YG-200, Yagami, Nagoya, Japan). Body mass index (BMI) was calculated as weight $(\mathrm{kg})$ by squared height $\left(\mathrm{m}^{2}\right)$. Waist circumference (WC) was measured at the umbilical level $(\mathrm{cm})$.

Among the metabolic risk factors for coronary disease, obesity was operationally defined according to the criteria of the Japanese Society for the Study of Obesity (Kanazawa et al., 2002): total obesity as BMI $\geq 25 \mathrm{~kg} / \mathrm{m}^{2}$, and central obesity as $\mathrm{WC} \geq 85 \mathrm{~cm}$. Hypertension was defined as systolic blood pressure $\geq 140 \mathrm{mmHg}$, and/or diastolic blood pressure $\geq 90$ $\mathrm{mmHg}$, and/or receiving current medication for this condition (Japanese Society of Hypertension Guidelines Subcommittee for the Management of Hypertension, 2001). Fasting plasma glucose $\geq 126 \mathrm{mg} / \mathrm{dl}$ and/or receiving current medication for this condition was the criterion for hyperglycemia (The Committee of the Japan Diabetes Society on the diagnostic criteria of diabetes mellitus, 2002). Following the Report of the Japan Atherosclerosis Society (2002), hypertriglyceridemia was defined as serum triglyceride $\geq 150$ $\mathrm{mg} / \mathrm{dl}$, hypercholesterolemia as total cholesterol $\geq 220 \mathrm{mg} /$ dl, hypo HDL-cholesterol as HDL cholesterol $<40 \mathrm{mg}$ / dl, hyper LDL-cholesterol as LDL cholesterol $\geq 140 \mathrm{mg} / \mathrm{dl}$, and/or receiving current medication for these conditions. Dyslipidemia was defined as the presence of any one of the four risks listed above (hypertriglyceridemia, hypercholesterolemia, hypo HDL-cholesterol, or hyper LDLcholesterol). A morbidity index was calculated as a sum of the scores of the following items (one point for each item): hypertension, hyperglycemia, and dyslipidemia.

A questionnaire about health-related behaviors, including personal data about education and immigration history for the Japanese-Brazilians, was completed by each participant. Participants were classified into three groups (J-J, JB-B, JB-J) by residence and according to their history of immigration. Those engaged in sports activity in a typical week for more than 3 hours per week were included in the 'regular sports activity' category, others were categorized as 'no regular sports activity'. Daily physical activity spent in a typical week was based on responses to questions about occupation (whether a participant's job was physically demanding), household activities (cleaning, gardening, cooking, and shopping), and transportation (bicycle or walking as transportation for moving around the city). All three items were assessed using a fourpoint scale (none, low, moderate, high). Those that reported no or low levels of daily activity were classified as 'low daily physical activity'. For eating habits, participants were asked to report their usual weekly intake of fish (fillet, sticks, sushi, and others), meat (hamburger, steak, roast beef, meatloaf, and others), fried snacks (dumplings, tempura, French fries, and others), fat spread (butter, margarine, mayonnaise, and others), sweets (cakes, cookies, pies, pastries, chocolate candies, ice cream, and others), and alcoholic beverages (wine, beer, liquor, Japanese sake, Brazilian cachaça, vodka, and others).

\section{Statistical analysis}

Chi-square tests were used for the analyses of differences between groups for the categorical variables, and post hoc Mantel-Haenszel tests were applied when necessary (for the comparison between each pair of groups). Age-adjusted analyses of covariance (ANCOVA) were used to analyse differences between groups for the continuous variables. Ageadjusted regression analyses were performed for WC with the sum of metabolic risk factors for coronary disease (morbidity index) for each group, and for the overall data (four models). Logistic regression analyses (model 1) were conducted using central obesity ( $85 \mathrm{~cm}$ as a WC cut-off point) as the dependent variable (presence $=1$, absence $=0$ ), including groups (native Japanese as the reference) and age (years) as the independent variables. Additionally, a second model was tested adjusting for the following lifestyle factors that were shown to significantly vary across groups: regular sports activity, daily physical activity, and eating habits (fish, meat, fried snacks, and fat spread). All statistical analyses were performed using SPSS 13.0 for Windows and statistical significance was set at $p<0.05$.

\section{Results}

As shown in Table 1, the majority of the Japanese-Brazilians (JB-J and JB-B groups) were from the second generation of Japanese descendants. An analysis comparing first, second, and third generations of Japanese descendants within the JB-J and JB-B groups did not find significant differences between generations with respect to mean values of WC (data not shown). Accordingly, data for three generations were pooled for each of the three subject groups. The level of education varied significantly among groups, with higher levels of education among the JB-B men. Because participants in the JB-J group were significantly younger than those in the other two groups, the analyses were adjusted for age.

Figure 1 confirmed significantly higher mean values of WC in the JB-B group $(90.3 \mathrm{~cm})$ compared with the J-J $(84.4 \mathrm{~cm})$ and the JB-J $(87.6 \mathrm{~cm})$. Among various metabolic risk factors (Table 2), the J-J group showed a lower prevalence of total obesity and dyslipidemia compared to the other groups. Hypertension and hyperglycemia did not differ significantly among groups. Absence of regular sports activity was significantly higher in the JB-J group; however, this group reported higher daily physical activity compared to the other groups. The J-J reported significantly more frequent consumption of fish and lower consumption of meat and other fatty food items compared to the JB-J and JB-B groups. Table 3 shows a significant $(p<0.001)$ correlation between $\mathrm{WC}$ and the morbidity index in each group and for the overall analyses.

Prevalence of central obesity was significantly higher in the 
Table 1 Characteristics of the participants

\begin{tabular}{|c|c|c|c|c|}
\hline & J-J & JB-J & JB-B & $\chi^{2}$ \\
\hline Participant, n & 104 & 178 & 108 & \\
\hline Age, mean (SD) & $58.2(12.0)^{*}$ & $48.7(6.8)$ & $57.7(10.1)^{*}$ & \\
\hline \multicolumn{5}{|c|}{ Japanese generation, n (\%) } \\
\hline First-generation & $104(100.0)$ & $7(3.9)$ & $20(18.5)$ & $16.97^{\dagger}(\mathrm{df}=2)$ \\
\hline Second-generation & $\ldots$ & $136(76.4)$ & $70(64.8)$ & \\
\hline Third-generation & $\ldots$ & $35(19.7)$ & $18(16.7)$ & \\
\hline \multicolumn{5}{|l|}{ Education, $\mathrm{n}(\%)$} \\
\hline Primary $(\leq 8$ yr) & $40(38.5)$ & $35(19.7)$ & $20(18.5)$ & $22.23^{\ddagger}(\mathrm{df}=4)$ \\
\hline Secondary (9-11 yr) & $34(32.7)$ & $68(38.2)$ & $26(24.1)$ & \\
\hline University ( $\geq 12$ yr) & $30(28.8)$ & $75(42.1)$ & $62(57.4)$ & \\
\hline
\end{tabular}

J-J, Native Japanese; JB-B, Japanese-Brazilian residing in Brazil; JB-J, Japanese-Brazilian residing in Japan.* Significantly different from JB-J $(p<0.001)$. ${ }^{\dagger}$ Significant difference in a comparison between JB-B and JB-J groups $(p<0.001)$. ${ }^{\ddagger}$ Significant difference among all groups $(p<0.001)$.

Table 2 Prevalence of metabolic risks and lifestyle factors among groups

\begin{tabular}{|c|c|c|c|c|c|}
\hline & $J-J(n=104)$ & JB-J $(n=178)$ & JB-B $(n=108)$ & \multirow{2}{*}{$\chi^{2}$} & \multirow{2}{*}{ post hoc } \\
\hline & $\mathrm{n}(\%)$ & n $(\%)$ & n $(\%)$ & & \\
\hline Total obesity & $34(32.7)$ & $87(48.9)$ & $52(48.1)$ & $8.12 *$ & $\dagger \dagger$ \\
\hline Hypertension & $55(52.9)$ & $89(50.0)$ & $54(50.0)$ & 0.23 & \\
\hline Hyperglycemia & $5(4.8)$ & $9(5.1)$ & $13(12.0)$ & 5.95 & \\
\hline Hypertriglyceridemia & $19(18.3)$ & $50(26.8)$ & $41(38.0)$ & $10.15^{*}$ & $\dagger$ \\
\hline Hypercholesterolemia & $29(27.9)$ & $69(38.8)$ & $20(18.5)$ & $13.84 *$ & $\S$ \\
\hline Hypo HDL-cholesterol & $5(4.8)$ & $20(11.2)$ & $41(38.0)$ & $48.75^{* *}$ & $\dagger \S$ \\
\hline Hyper LDL-cholesterol & $26(25.0)$ & $66(37.1)$ & $12(11.1)$ & $25.31 * *$ & $\dagger \dagger$ \\
\hline Dyslipidemia & $46(44.2)$ & $106(59.6)$ & $64(59.3)$ & $7.34 *$ & $t+$ \\
\hline No regular sports activity & $55(52.9)$ & $167(93.8)$ & $65(60.2)$ & $57.25 * *$ & $\dagger \dagger \S$ \\
\hline Low daily physical activity & $40(38.5)$ & $7(3.9)$ & $53(49.1)$ & $86.29 * *$ & $\dagger \uparrow \S$ \\
\hline \multicolumn{6}{|c|}{ Eating habits (frequency $>5$ times/wk) } \\
\hline Fish & $22(21.2)$ & $13(7.3)$ & $5(4.6)$ & $17.95^{* *}$ & $\dagger t$ \\
\hline Meat & $0(0.0)$ & $45(25.3)$ & $24(22.2)$ & $19.94 * *$ & $\dagger$ \\
\hline Fried snacks & $1(1.0)$ & $21(11.8)$ & $7(6.5)$ & $7.21 *$ & $t$ \\
\hline Fat spreads & $12(11.5)$ & $76(42.7)$ & $39(36.1)$ & $19.41 * *$ & $\dagger$ \\
\hline Sweets & $9(8.7)$ & $22(12.4)$ & $8(7.4)$ & 1.82 & \\
\hline Alcoholic beverages & $62(59.6)$ & $86(48.3)$ & $50(46.3)$ & 4.11 & \\
\hline
\end{tabular}

J-J, Native Japanese; JB-B, Japanese-Brazilian residing in Brazil; JB-J, Japanese-Brazilian residing in Japan. * $p<0.05 ; * * p<0.001 .^{\dagger}$ Difference between JJ and JB-B, ${ }^{+}$Difference between J-J and JB-J, ${ }^{\S}$ Difference between JB-B and JB-J. ${ }^{\circledR}$ Any one of the four risks listed above.

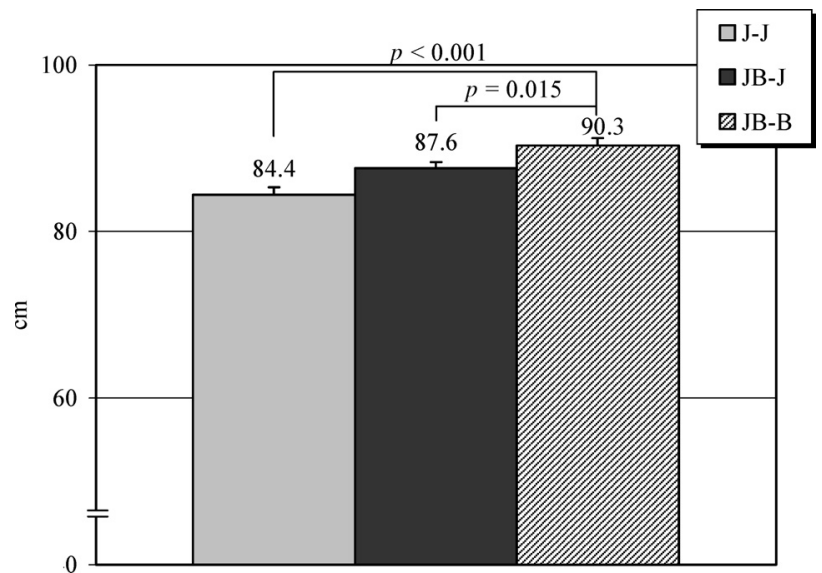

Fig. 1 Waist circumference among groups (age-adjusted mean and SE). J-J, Native Japanese; JB-B, JapaneseBrazilian residing in Brazil; JB-J, Japanese-Brazilian residing in Japan.
Table 3 Age-adjusted standard coefficients of a regression analyze for waist circumference values with morbidity index for coronary risk factors $^{\dagger}$

\begin{tabular}{lcc}
\hline & $\beta$ & $p$ \\
\hline J-J & 0.391 & $<0.001$ \\
JB-J & 0.337 & $<0.001$ \\
JB-B & 0.385 & $<0.001$ \\
Overall & 0.373 & $<0.001$ \\
\hline
\end{tabular}

J-J, Native Japanese; JB-B, Japanese-Brazilian residing in Brazil; JB-J, Japanese-Brazilian residing in Japan. ${ }^{\dagger}$ The sum of the scores of the following items (one point of each item): hypertension, hyperglycemia, and dyslipidemia (hypertriglyceridemia, or hypercholesterolemia, or hypo HDL-cholesterol, or hyper LDL-cholesterol).

JB-B (70.4\%) compared to the JB-J (56.2\%) and J-J (46.2\%) groups (Fig. 2). Furthermore, Table 4 confirmed the 2.8 -fold increased risk of developing central obesity in the JB-B group 


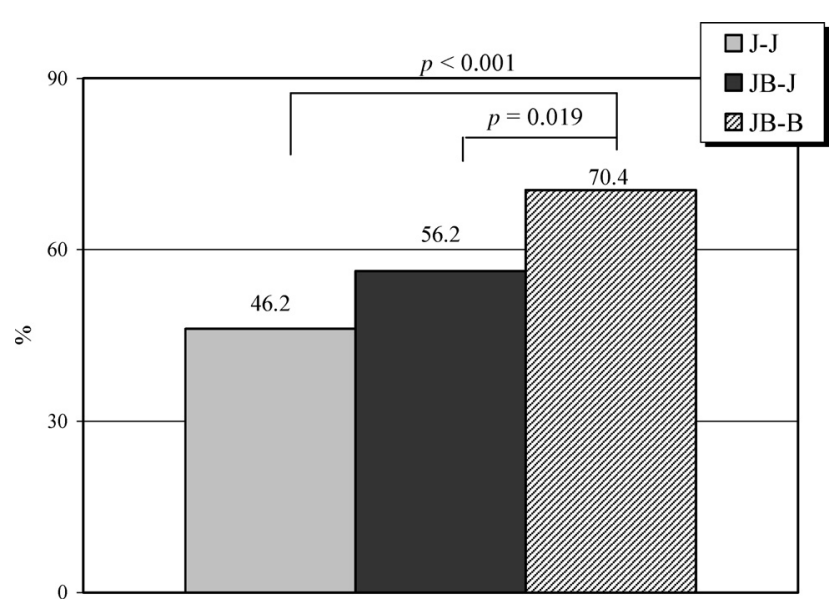

Fig. 2 Prevalence of central obesity $(\mathrm{WC}=85)$ among groups. J-J, Native Japanese; JB-B, Japanese-Brazilian residing in Brazil; JB-J, Japanese-Brazilian residing in Japan.

Table 4 Risk of developing central obesity among groups of Japanese ancestries

\begin{tabular}{lcrlll}
\hline & \multicolumn{2}{c}{ Model 1 $^{\dagger}$} & & \multicolumn{2}{c}{ Model 2 } \\
\cline { 2 - 3 } \cline { 5 - 6 } & Odds ratio (95\% CI) & $p$ & & Odds ratio (95\% CI) & $p$ \\
\hline J-J & 1 (ref) & & 1 (ref) & \\
JB-J & $1.56(0.91$ to 2.65$)$ & 0.104 & & $1.04(0.48$ to 2.27$)$ & 0.919 \\
JB-B & $2.81(1.59$ to 4.97$)$ & $<0.001$ & & $1.98(0.99$ to 4.00$)$ & 0.058 \\
\hline
\end{tabular}

Values are odds ratio and $95 \%$ confidence interval (CI). J-J, Native Japanese; JB-B, Japanese-Brazilian residing in Brazil; JB-J, JapaneseBrazilian residing in Japan. ${ }^{\dagger}$ Logistic regression model adjusted for age. ${ }^{\ddagger}$ Logistic regression model adjusted for age, regular sports activity, daily physical activity, and selected eating habit items (fish, meat, fried snacks, and fat spread).

compared to the J-J in the logistic model adjusted for age (model 1), whereas for the JB-J group it was not significant. After adjusting the logistic analyses for lifestyle factors (regular sports activity, daily physical activity, and consumption of fish, meat, fried snacks, and fat spread), the JB-B were found to be no longer significantly different in the model $(p=0.058)$.

\section{Discussion}

The present study has identified important and significant clinical differences in waist circumference and central obesity among the three population groups of Japanese descent, suggesting that immigration and cultural environment are associated with the pathogenesis of obesity. We have found that Japanese-Brazilians who live in Brazil (the JB-B group) have the highest waist circumference, native Japanese living in Japan (the J-J group) have the smallest waist circumferences, and Japanese-Brazilians who are living in Japan (JB-J) have intermediate waist circumferences. The prevalence of central obesity was found to be associated with increased levels of several metabolic risk factors that are known to be associated with coronary disease. Risk of developing central obesity was found to be higher in the JB-B group compared to the other groups, but the difference was no longer found to be significant once lifestyle factors were taken into account.

The increase in the prevalence of obesity is often ascribed to the changing lifestyle in westernized society, a common feature of which is the consumption of high-fat diets. A trend of change in the Japanese diet has been observed (World Health Organization, 2000b), where high consumption of animal fat and animal protein has increased rapidly (Matsumura, 2001). National survey revealed the contrasts of the diet of people living in Japan several decades ago, when $405 \mathrm{~g}$ of energy was consumed as carbohydrates and only $21.8 \mathrm{~g}$ as fat (Matsumura, 2001). The Japanese dietary pattern had changed considerably by 1996 , with consumption of $58.9 \mathrm{~g}$ of energy coming from fat (26.5\% of total energy) (Matsumura, 2001). Meanwhile, in Brazil, the traditional developing world problem of dietary malnutrition and undernutrition appears to be shifting rapidly to one of dietary excess. Concurrent increases in adult obesity have been observed among all groups of men and women with the greatest increase among lower income families (Monteiro et al., 1995). An increase in lipid content in the diet in less developed regions and of saturated fat in the country as a whole has been observed (Monteiro et al., 1995). This change is associated with a decrease in the consumption of beans, vegetables, fruits, and complex carbohydrates. Furthermore, there has been a simultaneous increase in sugar consumption from 1988 to 1996 in Brazil (Monteiro et al., 2000). These changes underscore the need for more public health initiatives in Brazil that are designed to increase public awareness of the importance of a healthy and nutritionally balanced diet.

Because all groups are originally from a similar Japanese genetic background, the differences in the prevalence of central obesity observed in this study are likely to be related to environmental or behavioral/lifestyle factors. It is well known that obesity is strongly associated with behavioral, cultural, educational, and social factors (Haslam and James, 2005; Paeratakul et al., 2002). This association is reflected in the findings of several migration studies that have shown increasing levels of obesity among populations that migrate (McDonald and Kennedy, 2005; Sundquist and Winkledy, 2000). Obesity has also been shown to be sensitive to the degree to which immigrants assimilate into the local culture (Amato et al., 2003; Klatsky and Armstrong, 1991). A number of lifestyle changes have been proposed as possible explanations for this association, including dietary factors, physical activity, and occupation choices among immigrants. Among the environmental changes impacting Japanese immigrants in Brazil, a study from Freire et al. (2005) suggested that changes in dietary habits were probably among the most important. The high proportion of fat in the Brazilian diet is known to contribute to the development of central obesity. Also, high 
amounts of protein intake have been shown to be associated with central obesity in Japanese-Brazilian living in São Paulo state (Ferreira et al., 2002). Likewise, our results showed higher consumption of meat and some fatty food items among Japanese-Brazilians.

Although lower education levels are frequently observed in developing countries, and have been associated with an increase risk for several diseases (Paeratakul et al., 2002; Paek and Hong, 2006), in our study, Japanese-Brazilians living in Brazil had higher education levels when compared to native Japanese. Indeed, other studies have also found that individuals of Japanese ancestry living in Brazil have relatively higher levels of education when compared to the general Brazilian population (Instituto Brasileiro de Geografia e Estatística, 2003). In our study, education levels were not found to be associated with increased risk of the development of obesity among Japanese-Brazilian living in Brazil.

Previous studies have reported a gradual increase in acceptance of the Western culture over time in Japanese immigrants, and an association between acculturation and an increased risk of developing metabolic disorders (Marmot and Syme, 1976; Miura et al., 2006; Tsugane et al., 1995; Yano et al., 1988). In our results, mean values of central obesity did not differ among first-, second-, and third-generations of JapaneseBrazilians. This fact was not widely explored in the present study, but we believe that it could be a consequence of the effect of globalization, and the rapid acculturation to local habits by the first-generation of Japanese immigrants to Brazil.

As we previously noted, central obesity (as indicated by WC) has been shown to be associated with an increased risk of coronary disease in men (Japan Society for the Study of Obesity, 2002; Kobayashi et al., 2001; Lakka et al., 2001; Zhu et al., 2005). Our findings are in agreement with this association, and support the use of WC as a simple vital sign that can serve as a predictor of morbidity among people of Japanese ancestry.

Regular sports activity is an important lifestyle behavior associated with improvements in both cardiorespiratory fitness and overall health status (Jakicic and Otto, 2006; Kay and Fiatarone Singh, 2006). A report from the World Health Organization (2002) identifies physical inactivity as the second most important risk factor for disease, after tobacco, highlighting its importance as a preventive strategy in people's daily lives. The native Japanese in our study were more engaged in regular sports activity, the Japanese-Brazilians residing in Japan were the least engaged, and the JapaneseBrazilians residing in Brazil were intermediate. However, those residing in Japan reported much higher levels of daily physical activity in their occupations, household activities, and transportation choices. These results suggest that the cultural environment in Japan may play a positive role in providing opportunities for daily physical activity among backimmigrant Japanese-Brazilians. In most cases, the underlying reason for back immigration to Japan was associated with economic issues. Occupation-related factors appear to be responsible for the higher levels of daily physical activity found in the Japanese-Brazilians in Japan. Employment in the JB-J group was mainly characterized by blue-collar activities, averaging 59.9-12.2 hours of work per week.

The global tobacco scenario shows that smoking is decreasing in most developed countries. In Japan, the smoking prevalence among men has declined considerably since 1966, when it peaked at 84\% (World Health Organization, 1997). The latest data on the prevalence of smoking among the male Japanese population was estimated of $45.9 \%$ (Ministry of Health, Labor and Welfare, 2003). Lung cancer became the leading cause of cancer deaths among Japanese men in 1993. Recently, lung cancer rates have continued to climb as the long-term consequences of post-World War II smoking popularity begin to take effect (World Health Organization, 2000 b). In Brazil, the proportion of smokers in the population dropped from approximately $32 \%$ to $18.8 \%$ between 1989 and 2003, and the annual per capita cigarette consumption decreased 42\% (Brazilian Ministry of Health, 2004). However, throughout Brazil, consumption trends among women and youngsters are of special concern, as tobacco companies have identified these groups as targets for their marketing strategies. In the present study, however, associations between tobacco and obesity were not investigated.

The results of the present study need to be interpreted within the context of certain limitations. First, participants were not randomly drawn from the Japanese population in Japan, or from the Japanese-Brazilian population in Japan and Brazil. Non-participants may have different characteristics and health status than the participants. Nonetheless, several studies have shown important evidence of the role of cultural environment on an immigrant's health and lifestyle using a similar recruiting procedure to that of the present study (Evenson et al., 2002; Klatsky and Armstrong, 1991; Kudo et al., 2000; Misra et al., 2005; Namekata et al., 1997; Parzaianello et al., 2005). Second, individuals in the JB-J group were significantly younger than those in the other two groups. Despite the use of similar recruiting procedures, and specific age inclusion criteria, it was not possible to equalize age across all groups. The disparity may have occurred due to the fact that the population of Japanese-Brazilians in Japan is composed mostly of economically active young adults. It is possible that this limitation may have underestimated the risk of developing central obesity in the JB-J. Moreover, although WC is strongly correlated with the amount of visceral fat, it is less precise in assessing visceral fat accumulation than computed tomography (CT) and magnetic resonance imaging (MRI). However, WC is frequently used in clinical and epidemiological studies, because of its good prediction of central obesity, low cost, and ease of measurement (Williams et al., 1997). Furthermore, WC is easy to measure in clinical settings and has the potential to serve as a "vital sign" which can predict the onset of obesityrelated conditions. An additional limitation to consider was that time spent in sports activity and daily physical activity was evaluated by non-standard methods. However, an advantage of 
our study was that we were able to directly measure all metabolic risk factors commonly associated with coronary disease.

In summary, we found substantial variation in measures of central obesity among the three groups of Japanese ancestry. Japanese-Brazilians residing in Brazil had the highest levels of central obesity. The variation between population groups in regard to central obesity illustrates the heterogeneity of risk factors among people of Japanese ancestry living in different cultural environments. Our data further suggest that a number of lifestyle choices may be important factors in the development of central obesity. Finally, there has been relatively little research on the health status of Japanese immigrants to Brazil. Further studies are necessary to explain the effects of different levels of acculturation on health-related factors across multiple generations of Japanese immigrants.

Acknowledgments This work was supported in part by the $21 \mathrm{st}$ century Center of Excellence (COE) program (2002-2007 Nishihira Project); Grants-in-Aid for Scientific Research (2003-2006 Tanaka Project) and doctoral fellowship (main author) from the Japanese Ministry of Education, Culture, Sports, Science and Technology; and a research fund (Bunka-Sumitomo Mitsui 2004) from the Brazilian Association of Japanese Culture. Deep thanks to all collaborators from the University of Tsukuba and São Paulo University. We greatly appreciate the participation of all community centers from Japan and Brazil, and to all the Japanese and Brazilians who voluntarily enrolled in this study.

\section{References}

Amato RV, Machado Cesar LA, Mansur AP, Hueb WA, Martins JRM, Vianna CB, Ramires JA (2003) Coronary heart disease clinical manifestation and risk factors in Japanese immigrants and their descendents in the city of Sao Paulo. Arq Bras Cardiol 81: 229-238

Brazilian Ministry of Health (2004) Brazil's ratification of the WHO framework convention on tobacco control: myths and truths. INCA, Rio de Janeiro

Cardoso MA, Hamada GS, Souza JM, Tsugane S, Tokudome S (1997) Dietary patterns in Japanese migrants to Southeastern Brazil and their descendants. J Epidemiol 7: 198-204

Curb JD, Marcus EB (1991) Body fat and obesity in Japanese Americans. Am J Clin Nutr 53: S1552-S1555

Evenson KR, Sarmiento OL, Macon LM, Tawney KW, Ammerman AS (2002) Environmental, policy, and cultural factors related to physical activity among latina immigrants. Women Health 36: 43-57

Ferreira SRG, Lerario DDG, Gimeno SGA, Sanudo A, Franco LJ (2002) Obesity and central adiposity in Japanese immigrants: role of the Western dietary pattern. J Epidemiol 12: $431-438$

Freire RD, Cardoso MA, Gimeno SGA, Ferreira SRG (2005)
Dietary fat is associated with metabolic syndrome in Japanese Brazilians. Diabetes Care 28: 1779-1785

Friedewald WT, Levy RI, Fredrickson DS (1972) Estimation of the concentration of low-density lipoprotein cholesterol in plasma, without use of the preparative ultracentrifuge. Clin Chem 18: 499-502

Gimeno SGA, Osiro K, Matsumura L, Massimino FL, Ferreira SRG (2005) Glucose intolerance and all-cause mortality in Japanese migrants. Diabetes Res Clin Pract 68: 147-154

Haslam DW, James WP (2005) Obesity. Lancet 366: 1197-1209

Hata Y, Mabuchi H, Saito Y, Itakura H, Egusa G, Ito H, Teramoto T, Tsushima M, Tada N, Oikawa S, Yamada N, Yamashita S, Sakuma N, Sasaki J, Working Committee on JAS Guideline for Diagnosis and Treatment of Hyperlipidemias (2002) Report of the Japan Atherosclerosis Society (JAS): Guideline for Diagnosis and Treatment of Hyperlipidemia in Japanese Adults. J Atheroscler Thromb 9: 1-27

Howarth NC, Murphy SP, Wilkens LR, Hankin JH, Kolonel LN (2006) Dietary energy density is associated with overweight status among 5 ethnic groups in the multiethnic cohort study. J Nutr 136: 2243-2248

Instituto Brasileiro de Geografia e Estatística (IBGE) (2003) Censo demográfico 2000, educação: resultado da amostra. IBGE [In Portuguese]

Iunes M, Franco LJ, Wakisaka K, Iochida LC, Osiro K, Hirai AT, Matsumura LK, Kikuchi M, Ferreira SR, Miyazaki N (1994) Self-reported prevalence of non-insulin-dependent diabetes mellitus in the 1st (Issei) and 2nd (Nisei) generation of Japanese-Brazilians over 40 years of age. Diabetes Res Clin Pract 24: S53-S57

Jakicic JM, Otto AD (2006) Treatment and prevention of obesity: what is the role of exercise? Nutr Rev 64: S57-S61

Japan Society for the Study of Obesity, The Examination Committee of Criteria for "Obesity Disease" in Japan (2002) New criteria for "obesity disease" in Japan. Circ J 66: 987-992

Japanese Society of Hypertension Guidelines Subcommittee for the Management of Hypertension (2001) Guidelines for the management of hypertension for general practitioners. Hypertens Res 24: 613-634

Kanazawa M, Yoshiike N, Osaka T, Numba Y, Zimmet P, Inoue S (2002) Criteria and classification of the obesity in Japan and Asia-Oceania. Asia Pacific J Clin Nutr 11: S732-S737

Kay SJ, Fiatarone Singh MA (2006) The influence of physical activity on abdominal fat: a systematic review of the literature. Obes Rev 7: 183-200

Klatsky AL, Armstrong MA (1991) Cardiovascular risk factors among Asian Americans living in Northern California. Am J Public Health 81: 1423-1428

Kobayashi H, Nakamura T, Miyaoka K, Nishida M, Funahashi T, Yamashita S, Matsuzawa Y (2001) Visceral fat accumulation contributes to insulin resistance, small-sized low-density lipoprotein, and progression of coronary artery 
disease in middle-aged non-obese Japanese men. Jpn Circ J 65: 193-199

Kudo Y, Falciglia GA, Couch SC (2000) Evolution of mean patterns and food choices of Japanese-American females born in the United States. Eur J Clin Nutr 54: 665-670

Kuzuya T, Nakagawa S, Satoh J, Kanazawa Y, Iwamoto Y, Kobayashi M, Nanjo K, Sasaki A, Seino Y, Ito C, Shima K, Nonaka K, Kadowaki T, The Committee of the Japan Diabetes Society on the diagnostic criteria of diabetes mellitus (2002) Report of the Committee on the classification and diagnostic criteria of diabetes mellitus. Diabetes Res Clin Pract 55: 65-85

Lakka TA, Lakka H, Salonen R, Kaplan GA, Salonen JT (2001) Abdominal obesity is associated with accelerated progression of carotid atherosclerosis in men. Atherosclerosis 154: 497-504

Marmot MG, Syme SL (1976) Acculturation and coronary heart disease in Japanese-Americans. Am J Epidemiol 104: 225-247

Matsumura Y (2001) Nutrition trends in Japan. Asia Pacific J Clin Nutr 10: S40-S47

McDonald JT, Kennedy S (2005) Is migration to Canada associated with unhealthy weight gain? Overweight and obesity among Canada's immigrants. Soc Sci Med 61: 2469-2481

McNeely MJ, Boyko EJ, Shofer JB, Newell-Morris L, Leonetti DL, Fujimoto WY (2001) Standard definition of overweight and central obesity for determining diabetes risk in Japanese Americans. Am J Clin Nutr 74: 101-107

Misra KB, Endemann SW, Ayer M (2005) Leisure time physical activity and metabolic syndrome in Asian Indian Immigrants residing in Northern California. Ethn Dis 15: 627-634

Ministry of Health, Labor and Welfare (2003) The national nutrition survey in Japan, 2001. Daiichi Shuppan, Tokyo

Miura K, Nakagawa H, Ueshima H, Okayama A, Saitoh S, Curb JD, Rodriguez BL, Sakata K, Okuda N, Yoshita K, Stamler J, INTERMAP Research Group, INTERLIPID Research Group (2006) Dietary factors related to higher plasma fibrinogen levels of Japanese-Americans in Hawaii compared with Japanese in Japan. Arterioscler Thromb Vasc Biol 26: 1674-1679

Miyatake N, Nishikawa H, Morishita A, Kunitomi M, Wada J, Suzuki H, Takahashi K, Makino H, Kira S, Fujii M (2002) Daily walking reduces visceral adipose tissue areas and improves insulin resistance in Japanese obese subjects. Diabetes Res Clin Pract 58: 101-107

Monteiro CA, Mondini L, Costa RBL (2000) Secular changes in dietary patterns in the metropolitan areas of Brazil (1988-1996). Rev Saúde Pública 34: 251-258

Monteiro CA, Mondini L, Medeiros AL, Popkin BM (1995) The nutrition transition in Brazil. Eur J Clin Nutr 49: 105-113

Nakanishi S, Okubo M, Yoneda M, Jitsuiki K, Yamane K, Kohno N (2004) A comparison between Japanese-
Americans living in Hawaii and Los Angeles and native Japanese: the impact of lifestyle westernization on diabetes mellitus. Biomed Pharmacother 58: 571-577

Namekata T, Moore D, Suzuki K, Mori M, Knopp R, Marcovina SM, Perrin EB, Hughes DA, Hatani S, Hayashi C (1997) Biological and lifestyle factors, and lipid and lipoprotein levels among Japanese Americans in Seattle and Japanese men in Japan. Int J Epidemiol 26: 1203-1213

Paek K, Hong Y (2006) Health behavior factors affecting waist circumference as an indicator of abdominal obesity. J Prev Med Pub Health 39: 59-66

Paeratakul S, Lovejoy JC, Ryan DH, Bray GA (2002) The relation of gender, race and socioeconomic status to obesity and obesity comorbidities in a sample of US adults. Int J Obes Relat Metab Disord 26: 1205-1210

Parzaianello L, Parzaianello NA, Coelho J (2005) Increased triglycerides levels in a Japanese population living in Southern Brazil. Arch Med Res 36: 59-64

Schrauwen P, Westerterp KR (2000) The role of high-fat diets and physical activity on the regulation of body weight. Br $\mathrm{J}$ Nutr 84: 417-427

Sundquist J, Winkledy M (2000) Country of birth, acculturation status and abdominal obesity in a national sample of Mexican-American women and men. Int $\mathrm{J}$ Epidemiol 29: 470-477

Tanaka S, Horimai C, Katsukawa F (2003) Ethnic differences in abdominal visceral fat accumulation between Japanese, African-Americans, and Caucasians: a meta-analysis. Acta Diabetol 40: S302-S304

Tsugane S, Hamada GS, Souza JM, Gotlieb SLD, Takashima Y, Todoriki H, Kabuto M, Karita K, Yamaguchi M, Watanabe S, Laurenti R (1994) Lifestyle and health-related factors among randomly selected Japanese residents in the city of São Paulo, Brazil, and their comparisons with Japanese in Japan. J Epidemiol 4: 37-46

Williams SRP, Jones E, Bell W, Davies B, Bourne MW (1997) Body habitus and coronary heart disease in men: a review with reference to methods of body habitus assessment. Eur Heart J 18: 376-393

World Health Organization (1997) Tobacco or Health: A Global Status Report. Country Profiles by Region. Available fromhttp://www.cdc.gov/tobacco/WHO/japan.htm. Accessed in 2 February 2007

World Health Organization (2000a) The Asia Pacific perspective: redefining obesity and its treatment. WHO Western Pacific Region, Australia

World Health Organization (2000b) World Health Organization issues new healthy life expectancy rankings, Japan number one in new 'healthy life' System, 2000. Available from http://www.who.int/inf-pr-2000/en/pr2000-life.html. Accessed in 2 February 2007

World Health Organization (2002) A physically active life through everyday transport, with a special focus on children and older people. WHO Regional Office for Europe

Yano K, MacLean CJ, Reed DM, Shimizu Y, Sasaki H, 
Kodama K, Kato H, Kagan A (1988) A comparison of the 12-year mortality and predictive factors of coronary heart disease among Japanese men in Japan and Hawaii. Am J Epidemiol 127: 476-487

Zhu S, Heymsfield SB, Toyoshima H, Wang Z, Pietrobelli A, Heshka S (2005) Race-ethnicity-specific waist circumference cutoffs for identifying cardiovascular disease risk factors. Am J Clin Nutr 81: 409-415
Received: August 26, 2006

Accepted: March 12, 2007

Correspondonce to: Kiyoji Tanaka, Graduate School of Comprehensive Human Sciences, Sports Medicine, University of Tsukuba, 1-1-1 Tennodai, D 616, Tsukuba, Ibaraki 305-8574, Japan

Phone: +81-29-853-2655

Fax: +81-29-853-6507

e-mail: tanaka@sports.taiiku.tsukuba.ac.jp andi@stat.taiiku.tsukuba.ac.jp 